\title{
Algebraic State Estimation for a Class of Switched Linear Systems
}

\author{
Yang TIAN ${ }^{1}$, Chengcheng SONG ${ }^{1}$, Wilfrid PERRUQUETTI ${ }^{2}$ \\ ${ }^{1}$ Sino-French Joint Laboratory of Automation and Signal Processing, \\ Automation School of Nanjing University of Science and Technology (NUST), \\ Nanjing, 210094, China, \\ tianyang@njust.edu.cn \\ ${ }^{2}$ LAGIS (CNRS, UMR 8219), Ecole Centrale de Lille (ECL), \\ Villeneuve d'Ascq, 59650, France \& Equipe Projet Non-A, INRIA Lille-Nord Europe, \\ wilfrid.perruquetti@ec-lille.fr
}

\begin{abstract}
In this paper, an algebraic state estimation method for a class of switched linear systems is derived. This approach is based on algebraic tools and distribution theory. Firstly, the unknown switching instant and the active mode are identified on-line, and then the process of state estimation is given by an explicit algebraic formula, rather than by an auxiliary dynamic system, which can be implemented formally and estimated very fast in computer. Numerical example and Simulations illustrate the efficiency of the proposed techniques.
\end{abstract}

Keywords: Algebraic approach, State estimation, Switching instants identification, Switched linear systems.

\section{Introduction}

Hybrid dynamical systems (HDS), in which continuous dynamics and discrete events coexist and interact between each other, can be used to model a large number of practical systems. Switched systems as higher--level abstractions of HDS, obtained by neglecting the details of the discrete behaviour. A family of dynamical linear subsystems and a switching law, which orchestrates the switching between them, can compose a switched linear system (see [1] for surveys).

In the recent years, there has been an increasing interest in the control problems of switched linear systems due to their significance from both a theoretical and practical point of view. Important results for switched systems have been achieved for problems of stability analysis [2], stabilization [3-5], tracking design [6] or controllability $[7,8]$.

Observability and state estimation is a very challenging problem for such systems since both the active mode and the continuous state have to be estimated during a finite time interval. The notion of state estimation for switched systems was firstly introduced in [9]. Observability notions for some classes of hybrid systems such as switched linear systems has been discussed and characterized in recent works such as $[1,10,11]$. The problem is to recover from available measurements the state of the system and/or the switching signal, and eventually the switching time. Different observation and identification methods have been performed during the last years [12-20]: state estimation for nonlinear switched system using Petri Net [12], for linear switched systems with unknown inputs [13], or an original and effective sampled and delayed output observer design which is based on hybrid switching systems [16] etc. Usually, the hybrid observer consists of two parts: an index estimator of the current active sub-model and a continuous observer that estimates asymptotically in most cases, the continuous state of the hybrid system.

The aim of this paper is to estimate the switching instants, active mode and the continuous state of a class of switched linear systems with the knowledge of the first active mode. The possibility to have finite time estimate for this kind of systems is clearly important. The approach considered here takes root in recent works developed in [21] for parameter identification of linear time-invariant systems. This method is based on algebraic tools (differential algebra, module theory and operational calculus) and results in finite time estimates given by explicit algebraic formula that can be implemented in a straightforward manner using standard tools from computational mathematics. Those results have been extended to the problems of closed-loop parametric estimation for continuous-time linear systems in [22], state estimation of linear 
systems in [23] or with time-varying parameters in [24], fault diagnosis in [25], nonlinear systems with unknown inputs in [26] or nonlinear feedback control in [27], switched systems estimation with Zeno phenomenon in [28]. This approach was also applied in [29] for the estimation of the index corresponding to the current active subsystem, and the state variable of this subsystem. Based on the result in [30, 31 ], finite time identification of the switching instants and the active mode are firstly studied, and the switching time estimation is given by an explicit formula, as a function of the integral of the output, in order to attenuate the influence of measurement noises. Then, combining our results of state estimation for linear time invariant systems by algebraic approach [23], we give the main approach of current active mode estimation and the continuous state estimation in real time.

This paper is organized as follows: Section 2 gives the problem statement and the mathematical formulation. The main result is derived in Section $3 \&$ Section 4. First, the switching instant identification of one commutation between two modes is analyzed. Then, the result is extended to the case of commutations among an arbitrary number of modes. In Section 4, with the estimated switching instants sequence and the algebraic state estimator for each mode ([23]), the state estimation of the autonomous switched linear systems is achieved. In section 5, simulation results that illustrate the proposed approach are provided. Finally, the last section is devoted to main conclusions and future works.

\section{Preliminary}

\subsection{Problem statement}

In this work, we study a class of switched linear systems called autonomous switched systems [1], i.e. the evolution of system determined by a collection of linear subsystems ( $Q$ modes) with continuous state connected by switches among a number of discrete state $q \in I_{Q} \triangleq\{1, \ldots, Q\}$, modelled by:

$\dot{x}=A_{q} x$,

where $x \in \mathbb{R}^{n}$ is continuous state, and $A_{q} \in \mathbb{R}^{n \times n}$ are constant matrices. For such a system, the switches are arbitrary and independent of the systems state variable. For the sake of convenience and without loss of generality, it is assumed that at each time $t \in \mathbb{R}$, only one discrete event acts on the system.

The necessary and sufficient conditions of the state observability of the autonomous switched linear systems (continuous state and the active mode) are derived in [1]. Note that the systems studied in this paper are assumed satisfied these conditions and observable. The objective is to estimate the state of the switched system (2.1) assuming that the first active mode $q$ is known. To do this, the crucial step is to estimate the unknown switching instant and the sequence of active modes. Hereafter, we will apply the algebraic method which involves high order time derivatives of $x(t)$. Due to the presence of non-smooth dynamics, derivation in this article has to be understood in the distribution sense.

\subsection{Mathematical formulation}

We recall here some standard definitions and results from distribution theory developed in [32], and fix the notations to be used. The space of $C^{\infty}$ - functions having compact support in an open subset $\Omega$ of $\mathbb{R}$ is denoted by $\mathcal{D}(\Omega)$, and $\mathcal{D}^{\prime}(\Omega)$ is the space of distributions on $\Omega$, i.e., the space of continuous linear functional on $\mathcal{D}(\Omega)$. For $u \in \mathcal{D}^{\prime},\langle u, \varphi\rangle$ denotes the real number which linearly and continuously depends on $\varphi \in \mathcal{D}$. This number is defined as $<u, \varphi>=\int_{-\infty}^{\infty} f \varphi$ for a locally Lebesgue integrable function $u=f$. The support of a distribution $u$, denoted as $\operatorname{supp} u$, is defined as the complement of the largest open subset of $\Omega$ in which the distribution $u$ vanishes.

For the Dirac distribution $u=\delta$ and its derivative $\dot{u}=\dot{\delta}$, the functional is defined as $\langle u, \varphi>=\varphi(0) \quad$ and $\quad\langle\dot{u}, \varphi>=-\dot{\varphi}(0)$, respectively. More generally, every distribution is indefinitely differentiable, by virtue of its definition:

$<\dot{u}, \varphi>=-<u, \dot{\varphi}>, \quad \varphi \in \mathcal{D}(\Omega)$.

In this paper, functions (locally Lebesgue integrable) are considered through the distributions they define, $\dot{u}$ or $u^{(1)}$ denote the distributional derivative of $u$, and $d u / d t$ stands for the distribution stemming from the 
usual derivative of $u$ defined almost everywhere. Hence, if $u$ is a continuous function except at point $a$ with a finite jump $\sigma_{a}$, its distributional derivative can be written as

$\dot{u}=d u / d t+\sigma_{a} \delta_{a}$,

where $\delta_{a}$ is the Dirac distribution located at point $\{a\}$. This result can be generalized to arbitrary derivation orders and discontinuity points as follows: let $\left\{t_{v}\right\}$ be an increasing sequence of points that are finite in every finite time interval. Assume that both left hand and right hand derivatives $\frac{d^{p} u}{d t^{p}}\left(t_{v}\right)$ exist and denote the corresponding jump $\sigma_{v}^{p}=\frac{d^{p} u}{d t^{p}}\left(t_{v}+\right)-\frac{d^{p} u}{d t^{p}}\left(t_{v}-\right)$. Then one has:

$u^{(p)}=\frac{d^{p} u}{d t^{p}}+\sum_{v} \sum_{k=0}^{p-1} \sigma_{v}^{p-1-k} \delta_{t_{v}^{(k)}}$.

In distribution sense, the class of differential equations we shall encounter will exhibit singular terms. The singularities, stemming from the origin $t_{0}=0$, will be gathered into a single distribution denoted $\psi_{0}$ with support $\{0\}$. The latter distribution $\psi_{0}$ will be referred to as initial condition term.

Generally, the multiplication of two distributions ( $\alpha$ and $u$ ) is well-defined when at least one of the two terms ( $\alpha$ here) is a smooth function. By definition:

$<\alpha u, \varphi>=<u, \alpha \varphi>$

With the properties (2.2), (2.4) and the reversed Leibniz rule, one can transform $\alpha u^{(n)}$ into linear combinations of derivatives of products $\alpha^{(k)} u$ :

$$
\alpha u^{(n)}=\sum_{k=0}^{n}(-1)^{2 n-k} C_{n}^{k} w_{k}^{(n-k)}, \quad w_{k}:=\alpha^{(k)} u
$$

Finally for every smooth function $\alpha$, one has:

$$
\alpha \delta_{\tau}=\alpha(\tau) \delta_{\tau},
$$

if there exists $\alpha^{(k)}(\tau)=0,(k=0, \cdots, r)$, then one has:

$$
\alpha \delta_{\tau}^{(r)}=0
$$

This nice property will be used later for the annihilation of singular distributions.

\section{Algebraic Switching Instant Estimation}

The goal of this section is to obtain an algebraic relation of the measured variables in order to explicitly estimate the unknown switching instants sequence $\left\{t_{j}\right\}_{j=1}^{\infty}$. Firstly, a state transformation is used to get an intermediate differential algebraic relation which parameters are not depending anymore on the switching instant but only on a Dirac distribution at this instant. Then, an annihilating algebraic manipulation based on (2.6) is provided to get the desired differential algebraic relation where the unknown switching instants explicitly appear. Finally, thanks to the integral operator, the expression of the switching instant is obtained in terms of time integrals of the state variables.

\subsection{The case of two modes and one commutation}

In this case, $q \in\{1,2\}$. Assume that the system (2.1) switches from one mode to the other one at instant $t_{c}$ and the continuous state of the active mode is known. (Note that the algebraic continuous state estimation will be detailed in Section 4.) Then, the dynamical behaviour of the system can be written as follows:

$$
\dot{x}=\Gamma(t) x, \quad \Gamma(t) \in\left\{A_{1}, A_{2}\right\} .
$$

Under the change of variable $z=e^{G t} x$ (constant matrix $G$ will be defined later), the system (3.1) is transformed as follow:

$\dot{z}=M(t) z$

with $M(t)=G+e^{G t} \Gamma(t) e^{-G t}$.

The matrix $G$ is chosen such that:

M_1 $(\mathrm{t})+\mathrm{M} \_2(\mathrm{t})=0$

with $M_{i}(t)=G+e^{G t} A_{i} e^{-G t}, \quad i=1,2$.

Since $G$ and $e^{G t}$ commute, equation (3.3) implies that $G=-\frac{A_{1}+A_{2}}{2}$ and

$\dot{z}=\sigma(t) M_{1}(t) z$ 
with $\sigma(t) \in\{-1,1\}$ and without loss of generality, $\sigma(0+)=1$ if $\Gamma(0+)=A_{1}$.

\section{Remark 1}

When matrices $A_{1}$ and $A_{2}$ commute, $M_{1}(t)=-M_{2}(t)=\frac{A_{1}-A_{2}}{2}$ are constant. This case was treated in [27]\}.

The determinant $\Delta_{1}(t)$ of $M_{1}(t)$ satisfies the following property:

$$
\begin{aligned}
& 2^{n} \Delta_{1}(t)=\operatorname{det}\left(2 M_{1}(t)\right)=\operatorname{det}\left(M_{1}-M_{2}\right) \\
& =\operatorname{det}\left(A_{1}-A_{2}\right)=\text { cte. }
\end{aligned}
$$

Assume that the matrix $\left(A_{1}-A_{2}\right)$ is full rank (If not, see [30] for the non-full rank case, the principle idea is to find an impulsive mode corresponds by algebraic operate, Lie algebras etc.). Then, $M_{1}(t)$ is invertible and one can define

$$
W_{1}(t):=\operatorname{Ad}\left(M_{1}(t)\right)=\Delta_{1}(t) M_{1}^{-1}(t),
$$

Then the equation (3.4) becomes

$$
W_{1}(t) \dot{z}=\sigma(t) \Delta_{1}(t) z
$$

Note that every term in (3.6) is known but the evolution of $\sigma(t)$. Since $\sigma(t)$ is a constant outside the set $\left\{t_{c}\right\}$, the distributional derivative of the product $\sigma(t) u(t)$ is well-defined when $u(t)$ is a smooth function.

Denoting $\sigma_{c}=\sigma\left(t_{c}+\right)-\sigma\left(t_{c}-\right)= \pm 2 \quad$ and using the properties (2.3) and (2.5), one has:

$$
\begin{aligned}
& \dot{\sigma}(t)=\sigma_{c} \delta_{t_{c}}, \\
& (\sigma(t) \dot{u}(t))=\dot{\sigma}(t) u(t)+\sigma(t) \dot{u}(t) \\
& =\sigma_{c} u\left(t_{c}\right) \delta_{t_{c}}+\sigma(t) \dot{u}(t)
\end{aligned}
$$

By derivation of (3.6) by using property (3.7), one obtains:

$$
W_{1}(t) \ddot{z}+\dot{W}_{1}(t) \dot{z}-\Delta_{1}(t) M_{1}(t) z=\dot{\sigma}(t) \Delta_{1}(t) z .
$$

Then the equation (3.8) can be rewritten as:

$$
\sum_{i=0}^{2} K_{i}(t) z^{(i)}=\gamma_{c} \delta_{t_{c}}
$$

with $K_{1}(t)=\dot{W}_{1}(t), \quad K_{0}(t)=-\Delta_{1}(t) M_{1}(t)$,

$$
K_{2}(t)=W_{1}(t) \text { and } \gamma_{c}=\sigma_{c} \Delta_{1}\left(t_{c}\right) z\left(t_{c}\right) \text {. }
$$

Thus, using the change of variables $z=e^{G t} x$, one obtains a differential system with a lefthand side that only contains known quantities. The right-hand side involves a Dirac distribution that will be annihilated using property (2.6), as shown in the next subsection.

\subsection{Explicit computation of the switching instant}

In this subsection, the estimation of switching instant is presented. The main idea is to identify the impulsive systems (3.9) based on two steps: (i) multiplication with smooth function $\alpha$ for the annihilation of singular distributions; (ii) integration by parts to express the switching instant. From the property (2.6), let's take an arbitrary smooth function $\alpha=f\left(t, t_{c}\right)$ with the following properties:

$$
f\left(t, t_{c}\right) \delta_{t_{c}}=0, f\left(0, t_{c}\right)=\dot{f}\left(0, t_{c}\right)=0 .
$$

Multiplying (3.9) by $f\left(t, t_{c}\right)$, one obtains:

$$
f\left(t, t_{c}\right)\left(\sum_{i=0}^{2} K_{i}(t) z^{(i)}\right)=0 .
$$

Integrating by parts (3.10) twice from 0 to $t>t_{c}$ and using the property

$\int_{0}^{t} \int_{0}^{t_{v-1}} \cdots \int_{0}^{t_{1}} x(\tau) d t_{v-1} \cdots d t_{1} d \tau=\int_{0}^{t} \frac{(t-\tau)^{v-1}}{(v-1) !} x(\tau) d \tau$,

one obtains:

$\int_{0}^{t}(t-\tau)\left(\ddot{f}\left(\tau, t_{c}\right) W_{1}(\tau)+\dot{f}\left(\tau, t_{c}\right) \dot{W}_{1}(\tau)-\Delta_{1}(\tau) M_{1}(\tau)\right) z(\tau) d \tau$

$+f\left(t, t_{c}\right) W_{1}(t) z(t)-2 \int_{0}^{t} \dot{f}\left(\tau, t_{c}\right) W_{1}(\tau) z(\tau) d \tau$

$-\int_{0}^{t} f\left(\tau, t_{c}\right) \dot{W}_{1}(\tau) z(\tau) d \tau=0$

So the estimation of $t_{c}$ can be obtained from (3.11). For example: $f\left(t, t_{c}\right)=t^{2}\left(t-t_{c}\right)$ which satisfies the properties of (i) and (ii).

Using the result of (3.11), the estimate of $t_{c}$ is given by the following formula:

$D\left(t, z, M_{1}\right) t_{c}=N\left(t, z, M_{1}\right)$

with 


$$
\begin{aligned}
& N\left(t, z, M_{1}\right)=t^{3} W_{1}(t) z(t)-\int_{0}^{t}\left(6 \tau^{2} W_{1}(\tau)+\tau^{3} \dot{W}_{1}(\tau)\right) z(\tau) d \tau \\
& +\int_{0}^{t}(t-\tau)\left(6 \tau W_{1}(\tau)+3 \tau^{2} \dot{W}_{1}(\tau)-\Delta_{1}(\tau) M_{1}(\tau)\right) z(\tau) d \tau \\
& D\left(t, z, M_{1}\right)=t^{2} W_{1}(t) z(t)-\int_{0}^{t}\left(4 \tau W_{1}(\tau)+\tau^{2} \dot{W}_{1}(\tau)\right) z(\tau) d \tau \\
& +\int_{0}^{t}(t-\tau)\left(2 W_{1}(\tau)+2 \tau \dot{W}_{1}(\tau)\right) z(\tau) d \tau
\end{aligned}
$$

Note that $N\left(t, z, M_{1}\right)$ and $D\left(t, z, M_{1}\right)$ are column vectors of dimension $n$. So one has

$t_{c}=\frac{N_{g}\left(t, z, M_{1}\right)}{D_{g}\left(t, z, M_{1}\right)}$,

for any $1 \leq g \leq n$, where $N_{g}\left(t, z, M_{1}\right)$ and $D_{g}\left(t, z, M_{1}\right)$ are the components of $N\left(t, z, M_{1}\right)$ and $D\left(t, z, M_{1}\right)$, respectively.

\section{Remark 2}

When the measured signal $z(t)$ is perturbed by noise, (3.11) can be integrated one or several more times in order to reduce the noise effect. In this case, the expression of $N\left(t, z, M_{1}\right)$ and $D\left(t, z, M_{1}\right)$ in (3.12) becomes

$$
\begin{aligned}
& N\left(t, z, M_{1}\right)=\int_{0}^{t} \frac{1}{2}(t-\tau)^{2}\left(6 \tau W_{1}(\tau)+3 \tau^{2} \dot{W}_{1}(\tau)-\Delta_{1}(\tau) M_{1}(\tau)\right) z(\tau) d \tau \\
& \quad+\int_{0}^{t} \tau^{3} W_{1}(\tau) z(\tau) d \tau-\int_{0}^{t}(t-\tau)\left(6 \tau^{2} W_{1}(\tau)+\tau^{3} \dot{W}_{1}(\tau)\right) z(\tau) d \tau \\
& D\left(t, z, M_{1}\right)=\int_{0}^{t} \frac{1}{2}(t-\tau)^{2}\left(2 W_{1}(\tau)+2 \tau \dot{W}_{1}(\tau)\right) z(\tau) d \tau \\
& \quad+\int_{0}^{t} \tau^{2} W_{1}(\tau) z(\tau) d \tau-\int_{0}^{t}(t-\tau)\left(4 \tau W_{1}(\tau)+\tau^{2} \dot{W}_{1}(\tau)\right) z(\tau) d \tau
\end{aligned}
$$

The method of switching instant identification is given above. Next, this method is extended to the general case.

\subsection{The case of $Q$ modes and $S$ commutations}

For each pair of matrices $A_{i}$ and $A_{j}\left(i, j \in I_{Q}\right.$ and $i \neq j$ ) in system (3.1), assume that $\left(A_{i}-A_{j}\right)$ is full rank. It has been seen that an estimator $E_{i, j}$ which computes the quantities $D\left(t, z, M_{i, j}\right)$ and $N\left(t, z, M_{i, j}\right)$, with the used calculation expressions defined as follows:

$$
M_{i, j}=G_{i, j}+e^{G_{i, j} t} A_{i} e^{-G_{i, j} t}, \quad G_{i, j}=-\frac{A_{i}+A_{j}}{2}
$$

and these can be used to determine a switching instant $t_{i, j}$ that occurs between the mode $i$ and $j$ (either from mode $i$ to mode $j$ or from $j$ to mode $i$ ). Hence, in order to identify all the switches among $Q$ modes, one can use $C_{Q}^{2}=(Q(Q-1)) / 2$ estimators in parallel. Then, the output signals of each estimator can determine the occurrence of a switch and its associated modes. Furthermore, with the knowledge of the first active mode, the whole sequence of active mode can be identified.

\section{Algebraic State Estimation}

An algebraic online switching instant estimation of the linear switched system is introduced in Section 3, this section is devoted to the estimation of the active mode and the continuous state of this class of system.

Hereafter, the system modelled by (2.1) and satisfying the following constraints is studied:

- Each mode is observable;

- The initial mode $i$ from which the system starts is known;

The matrix $\left(A_{i}-A_{j}\right)$ is full rank for each pair of matrices $A_{i}, A_{j}(i \neq j)$ or each pair of matrices $A_{i}$ and $\mathrm{A}_{j}$ commute (if not, see [30] to treat the non-full rank case ).

The main steps of the proposed method are:

1) Estimate the continuous state variables $x_{1}, \ldots, x_{n}$ of the mode $i$ with algebraic approach presented in article [23] knowing that the initial mode is $i$. The states of the other modes are estimated in parallel in order to follow the trajectory of the state faster when switch occurs.

2) Estimation of the switching instant and the active mode $j$ with the method proposed in section 3 .

3) Once the index $j$ of the current active mode is known, estimation the current state variable of the system $\rightarrow$ step 1 .

\section{Numerical Example}

Consider an autonomous switched system in continuous time which commutes among three 
linear time-invariant modes described by the following defined matrices:

$$
\begin{aligned}
& A_{1}=\left(\begin{array}{ll}
1 & 1 \\
0 & 1
\end{array}\right), A_{2}=\left(\begin{array}{ll}
3 & 2 \\
0 & 3
\end{array}\right), \quad A_{3}=\left(\begin{array}{ll}
2 & 1 \\
5 & 0
\end{array}\right), \text { and } \\
& C=\left(\begin{array}{ll}
1 & 0
\end{array}\right) .
\end{aligned}
$$

\subsection{Continuous state estimation}

Firstly, we obtain an explicit formulation of the output estimation $y_{e}$ and its derivative $\dot{y}_{e}$ of each mode using the method proposed in [23]. Secondly, since each mode is observable then their observability matrix is invertible. Thus, it is possible to express the state in function of the output and its derivative. By replacing this relationship with the estimated values, we can reconstruct state variable in function of the integral of $y(t)$.

For mode $A_{1}$, one has:

$$
\begin{aligned}
& \left\{\begin{array}{l}
y_{e}(t)=\frac{6 \int_{0}^{t} \tau y(\tau) e^{(t-\tau)} d \tau-2 t \int_{0}^{t} y(\tau) e^{(t-\tau)} d \tau}{t^{2}} \\
\dot{y}_{e}(t)=\frac{6 \int_{0}^{t} \tau y(\tau) e^{(t-\tau)} d \tau-2 t \int_{0}^{t} y(\tau) e^{(t-\tau)} d \tau-2 \int_{0}^{t} y(\tau) e^{(t-\tau)} d \tau}{t^{2}}
\end{array}\right. \\
& \left\{\begin{array}{l}
x_{1 e}=y_{e} \\
x_{2 e}=\dot{y}_{e}(t)-y_{e}(t)
\end{array}\right.
\end{aligned}
$$

For mode $A_{2}$, one has:

$$
\begin{aligned}
& y_{e}(t)=\frac{6 \int_{0}^{t} \tau y(\tau) e^{3(t-\tau)} d \tau-2 t \int_{0}^{t} y(\tau) e^{3(t-\tau)} d \tau}{t^{2}} \\
& \dot{y}_{e}(t)=\frac{18 \int_{0}^{t} \tau y(\tau) e^{3(-\tau)} d \tau-6 t \int_{0}^{t} y(\tau) e^{3(t-\tau)} d \tau-2 \int_{0}^{t} y(\tau) e^{3(1-\tau)} d \tau}{t^{2}}+\frac{2 y_{e}(t)}{t} \\
& \left\{\begin{array}{l}
x_{1 e}=y_{e}(t) \\
x_{2 e}=\frac{1}{2}\left(\dot{y}_{e}(t)-3 y_{e}(t)\right)
\end{array}\right.
\end{aligned}
$$

For mode $A_{3}$, one has:

$$
\left\{\begin{array}{l}
y_{e}(t)=\frac{2 \int_{0}^{t}\left(e^{(1-\sqrt{6})(t-\tau)}+e^{(1+\sqrt{6})(t-\tau)}\right) \tau y(\tau) d \tau}{t^{2}} \\
-\frac{\sqrt{6} 6 \int_{0}^{t}\left(e^{(1+\sqrt{6})(t-\tau)}-e^{(1-\sqrt{6})(t-\tau)}\right) y(\tau) d \tau}{t^{2}} \\
\dot{y}_{e}(t)=\frac{\frac{6-6 \sqrt{6}}{3} \int_{0}^{t} e^{(1-\sqrt{6})(t-\tau)} \tau y(\tau) d \tau+\frac{6+6 \sqrt{6}}{3} \int_{0}^{t} e^{(1+\sqrt{6})(t-\tau)} \tau y(\tau) d \tau}{t^{2}} \\
-\frac{\left(\frac{6-\sqrt{6}}{6} \int_{0}^{t} e^{(1-\sqrt{6})(t-\tau)} y(\tau) d \tau+\frac{6+\sqrt{6}}{6} \int_{0}^{t} e^{(1+\sqrt{6}(t-\tau)} y(\tau) d \tau\right)}{t^{2}}+\frac{2 y_{e}(t)}{t}
\end{array}\right.
$$

$\left\{\begin{array}{l}x_{1 e}=y_{e}(t) \\ x_{2 e}=\dot{y}_{e}(t)-2 y_{e}(t)\end{array}\right.$

\subsection{Numerical simulation results}

The switching instant is assumed to occur at the following instants of

$t_{1}=0.4 \mathrm{~s}, t_{2}=1 \mathrm{~s}, t_{3}=1.2 \mathrm{~s}, t_{4}=1.4 \mathrm{~s}$ and $t_{5}=1.5 \mathrm{~s}$ (cf. Fig. 1, Fig. 2) and the initial mode is mode 1 . Their corresponding switching sequence is designed as follows

$$
A_{1} \rightarrow A_{3} \rightarrow A_{2} \rightarrow A_{1} \rightarrow A_{3} \rightarrow A_{2} \text { (cf. Fig. 3). }
$$

Since estimator $1\left(E_{1,2}\right)$ detects the switching which occurs between the mode $A_{1}$ and the mode $A_{2}$, the estimator $2\left(E_{2,3}\right)$ corresponds to commutation between mode $A_{2}$ and mode $A_{3}$, the estimator $3\left(E_{1,3}\right)$ detects the commutation between the mode $A_{1}$ and the mode $A_{3}$, it is known that estimator $3 \rightarrow 2 \rightarrow 1 \rightarrow 3 \rightarrow 2$ works respectively (cf. Fig. 4).

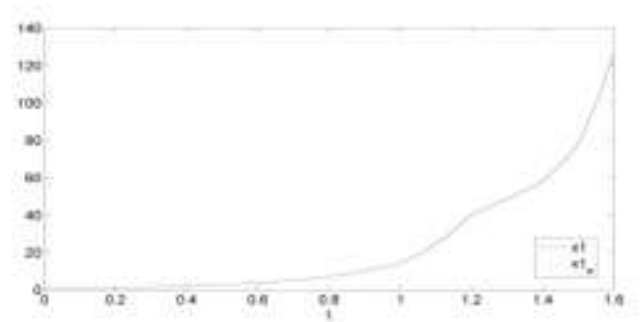

Figure 1. Real and estimated state value: $x_{1}$.

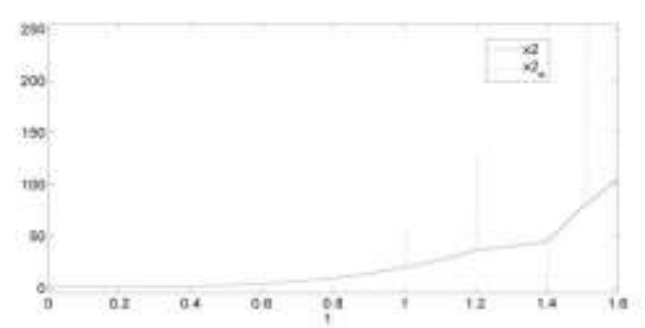

Figure 2. Real and estimated state value: $x_{2}$.

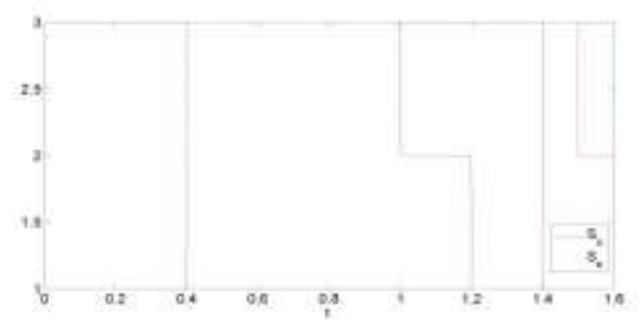

Figure 3. Real and estimated active mode $S_{o} \& S_{e}$. 


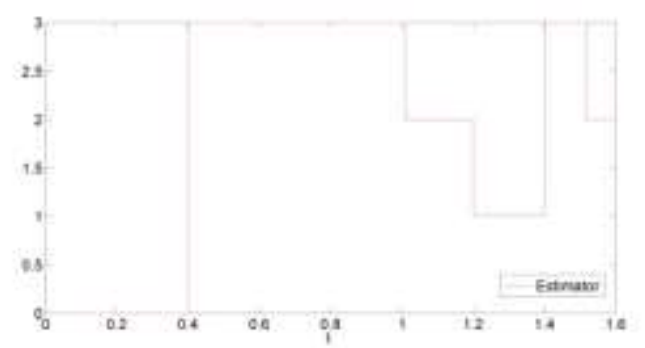

Figure 4. Sequences of the used estimator.

\section{Conclusion}

In this paper, an algebraic identification of switching instant, active mode and the continuous state estimation of autonomous switched linear systems has been introduced. Based on algebraic tools and distribution theory, an explicit algorithm computes the switching instants has been derived, then combining our previous results of algebraic state estimation for linear systems, the active mode and continuous state estimation in real time are given. Future works are concerned with the application to the fault diagnosis and state estimation of a class of the switched systems with time-varying modes.

\section{Acknowledgements}

This work was partially supported by the National Natural Science Foundation of China (61304077, 61203115), by the Natural Science Foundation of Jiangsu Province (BK20130765), by the Specialized Research Fund for the Doctoral Program of Higher Education of China (20123219120038), by the Chinese Ministry of Education Project of Humanities and Social Sciences (13YJCZH171), by the Fundamental Research Funds for the Central Universities (30920130111014), and by the Zijin Intelligent Program of Nanjing University of Science and Technology (2013_ZJ_0105).

\section{REFERENCES}

1. VIDAL, R., A. CHIUSO, S. SOATTO, S. SASTRY, Observability of Linear Hybrid Systems. Lect. Notes in Comp. Sc. (Hybrid Systems Computation and Control). vol. 2623, 2003, pp. 526-539.

2. AGRACHEV, A. A., D. LIBERZON, Liealgebraic Stability Criteria for Switched Systems, SIAM J. Ctrl Opt., vol. 40, 2001, pp. 253-269.
3. SOGA, T., N. OTSUKA, Stabilizability Conditions for Switched Linear Systems with Constant Input via Switched Observer, Studies in Informatics and Control, vol. 22, no. 1, 2013, pp. 7-14.

4. ZHAI G., H. LIN, P.J. ANTASAKLIS, Quadratic Stabilizability of Switched Linear Systems with Polytopic Uncertainties. Intl. Journal of Control, vol. 76, no. 7, 2003, pp. 747-753.

5. WICKS, M., P. PELETIES, R. DECARLO, Switched Controller Synthesis for the Quadratic Stabilisation of a Pair of Unstable Linear Systems. European J. of Control, vol.4, no. 2, 1998, pp.140-147.

6. BOURDAIS, R., M. FLIESS, C. JOIN et al Towards a Model-free Output Tracking of Switched Nonlinear Systems. 7th IFAC Symp. on Nonlinear Control Systems, 2007.

7. SUN, Z, S. S. GE, Analysis and Synthesis of Switched Linear Control Systems. Automatica, vol.41, no.2, 2005, pp.181-195.

8. XIE G, D. ZHENG, L. WANG, Controllability of Switched Linear Systems. IEEE Trans. on Aut. Ctrl, vol. 47, no.8, 2002, 1401-1405.

9. ACKERSON, G. A., K. S. FU, On State Estimation in Switching Environments. IEEE Trans. on Aut. Ctrl. 15(1), 1970, pp. 1017.

10. BABAALI, M., G. J. PAPPAS, Observability of Switched Linear Systems in Continuous time. Hybrid Syst. Comp. and Control. Springer Berlin Heidelberg, 2005, pp. 103-117.

11. De SANTIS, E., M. D. DI BENEDETTO, G. POLA, On Observability and Detectability of Continuous-time Linear Switching Systems. 42nd IEEE Conf. Dec. \& Ctrl, 2003.

12. ARICHI, F., M. DJEMAI, B. CHERKI et al. State Estimation for a Class of Nonlinear Switched Systems using Petri Net. 2nd Intl. Symp. IEEE Envir. Friendly Energies \& App. (EFEA), 2012, pp. 38-43.

13. RIOS, H., J. DAVILA, F. LEONID et al. State Estimation for Linear Switched Systems with Unstable Invariant Zeros and Unknown Inputs. 51st IEEE Conf. on Decision and Control, USA, 2012.

14. WANG, H. P., C. VASSEUR, V. KONCAR, A. CHAMROO, N. CHRISTOV, Design 
and Implementation of Robust Hybrid Control of Vision based Underactuated Mechanical Nonminimum Phase Systems, Studies in Informatics and Control, vol.19, no.1, 2010, pp. 35-44.

15. LI, S. Z., H. P. WANG, Y. TIAN, N. CHRISTOV, A. AITOUCHE, NNPID based Stator Voltage Oriented Vector Control for DFIG, Studies in Informatics and Control, vol. 23, no.1, 2014, pp. 5-12.

16. WANG, H. P., C. VASSEUR, N. CHRISTOV, V. KONCAR, Vision Servoing of Robot Systems Using Piecewise Continuous Controllers and Observers, Mech. Syst. and Sign. Proc., vol.33, 2012, pp. 132-141.

17. WANG, H. P., N. CHRISTOV, EventTriggered Observer based Control of Networked Visual Servoing Control Systems, Control Engineering and Applied Informatics, vol.16, no.1, 2014, pp. 22-30.

18. ALESSANDRI, A., P. COLETTA, Design of Luenberger Observers for a Class of Hybrid Linear Systems. Hybrid Systems: Comp. \& Ctrl, Springer Verlag. vol. 2034, 2001, pp. 7-18.

19. BALLUCHI, A., L. BENVENUTI, M. D. D. BENEDETTO, A. L. SANGIOVANNIVINCENTELLI, Design of Observers for Hybrid Systems. Lect. Notes in Computer Science, Springer Verlag, 2002, pp. 77-89.

20. BOUBAKER, S., M. DJEMAI, N. MANAMANNI et al. Active Modes and Switching Instants Identification for Linear Switched Systems based on Discrete Particle Swarm Optimization. App. Soft Comp. vol.14, 2014, pp. 482-488.

21. FLIESS, M., H. SIRA-RAMIREZ, An Algebraic Framework for Linear Identification. ESAIM Ctrl. Optim. Calc. Variat., vol. 9, 2003, pp. 151-168.

22. FLIESS, M., H. SIRA-RAMIREZ, Closedloop Parametric Identification for Continuous-time Linear Systems via New Algebraic Techniques. Cont.-Time Model Ident. from Sampled Data. Springer, 2008.
23. TIAN, Y., T. FLOQUET, W. PERRUQUETTI, Fast State Estimation in Linear Time-invariant Systems: An Algebraic Approach. 16th Medit. Conf. Contr. Automat., Corsica, France, 2008.

24. TIAN, Y., T. FLOQUET, W. PERRUQUETTI, Fast State Estimation in Linear Timevarying Systems: An Algebraic Approach, 47th IEEE Conf. on Dec. \& Ctrl, Mexico, 2008.

25. FLIESS M., C. JOIN, H. SIRA-RAMIREZ, Robust Residual Generation for Linear Fault Diagnosis: An Algebraic Setting with Examples. Intl. J. of Control, vol.77, no.14, 2004, pp. 1223-1242.

26. BARBOT, J. P., M. FLIESS, T. FLOQUET, An Algebraic Framework for the Design of Nonlinear Observers with Unknown Inputs. 46th IEEE Conf. on Decision and Control, New Orleans, 2007.

27. MBOUP, M., C. JOIN, M. FLIESS, A Revised Look at Numerical Differentiation with an Application to Nonlinear Feedback Control. 15th IEEE Medit. Conf. on Ctrl and Auto., 2007.

28. ZHENG G., L. YU, D. BOUTAT et al. Algebraic Observer for a Class of Switched Systems with Zeno Phenomenon. 48th IEEE Conf. on Dec. and Control, 2009.

29. FLIESS M., C. JOIN, W. PERRUQUETTI, Real-time Estimation for Switched Linear Systems. 47th IEEE Conf. on Dec. and Control, 2008.

30. MINCARELLI, D., T. FLOQUET, L. BELKOURA, Active Mode and Switching Time Estimation for Switched Linear Systems. $50^{\text {th }}$ IEEE Conf. on Dec. and Control and ECC, Orlando, 2011.

31. TIAN, Y., T. FLOQUET, L. BELKOURA et al., Algebraic Switching Time Identification for a Class of Linear Hybrid Systems. J. of. Nonlinear Analysis: Hybrid systems, vol. 5, 2011, pp. 233-241.

32. SCHWARTZ, L., Theorie des distributions. 2nd Ed. Hermann, 1966. 